\title{
Is 5-Minute Heart Rate Variability a Useful Measure for Monitoring the Autonomic Nervous System of Workers?
}

\author{
Kyoung Bok MiN,,${ }^{1,2 \dagger} \mathrm{MD}$, Jin-Young Min, ${ }^{1 \dagger} \mathrm{PhD}$, Domyung PAEK, ${ }^{1} \mathrm{MD}$, \\ Sung-Il CHO, ${ }^{1} \mathrm{MD}$, and $\mathrm{Mia}$ Son, ${ }^{3} \mathrm{MD}$
}

\begin{abstract}
SUMMARY
Heart rate variability (HRV) is a noninvasive physiological marker used to assess autonomic nervous function and can be recorded over the short or long term. Long-term recording is a good method for assessing mortality and patient prognosis, while short-term measurement is widely used due to practical advantages and reproducibility. However, little is known about whether a short-term assessment reflects the variation in the overall heart rate of workers. This study evaluated the relationship between the 24-hour and 5minute HRV, which was selected from a 24-hour recording. The study population was 153 male workers at the National Rail Company in Korea, who had their heart rates assessed for 24 hours. In the time and frequency domains, the correlations of the HRV between 24 hours and 5 minutes were calculated for the entire time and limited times (09:00-17:00). We found modest correlations in the time $(\mathrm{R}=0.614-0.668)$ and frequency $(\mathrm{R}=0.508-0.817)$ domains, but the best correlation was for the high-frequency spectra (HF; $\mathrm{R}=0.817$ ). Our findings suggest that the short-term HRV remains stable and may be applicable for screening the variation in the heart rate of workers, although not all of the correlations were sufficiently strong. (Int Heart J 2008; 49: 175-181)
\end{abstract}

Key words: Cardiac autonomic function, Heart rate variability, Short-term, Long term, Workers

HEART rate variability (HRV) is a physiological maker used to assess the autonomic nervous system (ANS) and is significantly associated with a variety of pathologies, such as cardiovascular disease and diabetes mellitus. ${ }^{1-6)}$ The HRV can be assessed over the short (usually 5-15 minutes) or long (24 hours) term. Long-term measurements reflect the overall change in the heart rate under nonspecific, changing conditions because the subjects perform their usual daily activities during the test. The long-term method has been used to assess mortality and the adverse prognosis of patients on bed rest. ${ }^{7,8}$ This analysis is difficult and not

From the ${ }^{1}$ School of Public Health and Institute of Health and Environment, Seoul National University, Seoul, ${ }^{2}$ Department of Occupational and Environmental Medicine, Ajou University School of Medicine, Suwon, and ${ }^{3}$ Department of Preventive Medicine, School of Medicine, Kangwon National University, Gangwon-do, Republic of Korea.

${ }^{\dagger}$ Authors that contributed equally to this study.

Address for correspondence: Mia Son, MD, Department of Preventive Medicine, School of Medicine, Kangwon National University, Hyoja-dong, Chuncheon-si, Gangwon-do, 200-701, Republic of Korea.

Received for publication September 18, 2007.

Revised and accepted February 8, 2008. 
very reproducible. ${ }^{9)}$ Conversely, short-term measurements offer more practical advantages, including easy application in a clinical setting and a simplified data process, although short-term recording depends on the test conditions because the subjects are in a controlled environment. ${ }^{10)}$

Several studies have demonstrated that the 5-minute HRV, versus a 24-hour measurement, is a strong indicator of cardiac events in the normal population and patients, ${ }^{11-14)}$ and many have focused on predicting or evaluating the accuracy and reproducibility of measuring the 5-minute HRV. ${ }^{15,16)}$ Although we agree that the 5-minute HRV has good reproducibility and is more practical for application, it is not known whether a 5-minute assessment can replace the variation in the overall heart rate for monitoring and screening the ANS of workers. This study evaluated the relationship between the 24-hour and 5-minute HRV, which was selected from 24-hour recordings.

\section{METHODS}

Study population: The study population consisted of 153 male employees, aged 22-69 years, of the National Railway Company in Seoul, Korea. None were taking medicine that affected the ANS, or had a history of cardiac disease. The subjects completed a questionnaire that considered personal and work characteristics, and had their heart rate monitored for 24 hours. All subjects provided written informed consent before participating in this investigation.

Measurement of heart rate variability: HRV was assessed using a 3-channel SEER MC, 12-lead Ambulatory ECG recorder (Marquette Medical Systems, Milwaukee, WI, USA). The HRV was measured in all subjects for 24 hours while performing their usual daily activities. The recordings were analyzed using a Marquette $8000 \mathrm{~T}$ system (Marquette Electronics, Milwaukee, WI, USA), and screened to eliminate data artifacts. A technician trained at HRV assessment edited the analysis results and divided each into 288 (= 24 hour $\times 12), 5$-minute segments sampled from the 24-hour recordings.

Time- and frequency-domain indices were chosen for the HRV analysis, and consisted of 4 components: the standard deviation of the NN intervals (SDNN) and the average standard deviation of all $\mathrm{NN}$ intervals (ASDNN), the high frequency (HF) spectra $(0.15-0.40 \mathrm{~Hz})$, the low frequency (LF) spectra $(0.04-0.15$ $\mathrm{Hz}$ ), and the low/high frequency (LF/HF) ratio.

Statistical analysis: The following procedures were used in the analysis (Figure), and the statistical analysis was performed using SAS statistical software. ${ }^{17)}$ (1) Time-domain analysis. We calculated the correlation between the ASDNN of the 24-hour HRV and the SDNN of the 5-minute HRV, which was selected randomly from the 24-hour recordings. Also, when sampling 5-minute segments 

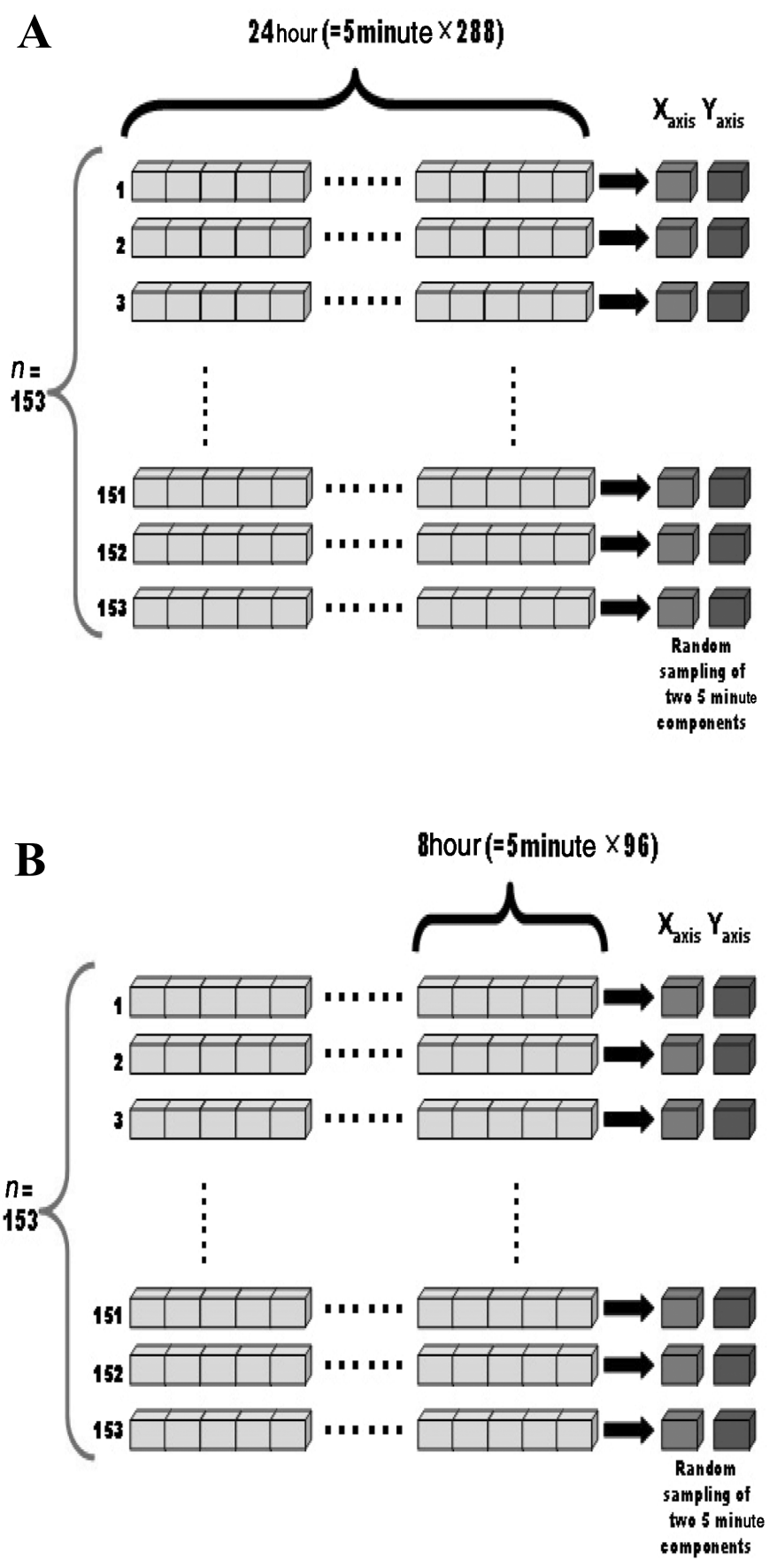

Figure. Description of statistical analysis according to 24-hour and office-hours measurement. A: 24-hour measurement. B: Office-hours measurement. 
from the 24-hour recordings, we limited the sampling time to between the hours of 09:00 and 17:00, and the association between the ASDNN and SDNN was then assessed, since HRV data are usually collected during standard office hours. These two analysis methods (24-hour measurement versus office hour measurement) were repeated randomly 41,328 times $\left(\mathrm{N}={ }_{288} \mathrm{C}_{2}=41,328\right)$ each, and the results were expressed as the mean value of Pearson's correlation coefficient.

(2) Frequency domain analysis. We randomly selected 5-minute segments from the 24-hour recording of each subject. Pearson's correlation coefficient was calculated to describe the frequency-domain index (HF and LF) between the 24-hour HRV and 5-minute HRV. The sampling time of the 5-minute segments and the 24hour HRV was restricted to between 09:00 and 17:00, and the association between the two variables was then assessed. Each analysis was performed 41,328 times $\left(\mathrm{N}={ }_{288} \mathrm{C}_{2}=41,328\right)$. The results were expressed as the mean value of Pearson's correlation coefficient.

\section{RESULTS}

The general characteristics of the subjects are described in Table I. The mean age of the subjects was 38.5 years, and their height and weight averaged $166.9 \pm 5.8 \mathrm{~cm}$ (mean $\pm \mathrm{SD}$ ) and $62.6 \pm 7.9 \mathrm{~kg}$, respectively. The means of the 4 components were calculated for the HRV indices. The mean SDNN was $56.9 \pm$ 18.0, and in the frequency-domain analysis, the LF, HF, and LF/HF ratios were $637.9,194.0$, and 5.7, respectively.

To account for the correlation between the 24-hour and 5-minute HRV, we evaluated 4 components of the time and frequency domains (Table II). For timedomain spectra, the correlations with SDNN and ASDNN were modest $(\mathrm{R}=$ 0.614 and 0.668 ), and the measurement during office hours gave a slightly higher correlation than the selection from the 24-hour HRV. For the frequency-domain analysis, the 3 components had correlation coefficients $(\mathrm{R}=0.508-0.817)$ similar

Table I. General Characteristics of the Subjects and the Heart Rate Variability $(n=153)$

\begin{tabular}{lrcc}
\hline & Mean & SD & Range \\
\hline Age (years) & 38.5 & 9.24 & $(22.1-59.1)$ \\
Height $(\mathrm{cm})$ & 166.9 & 5.8 & $(160.0-180.0)$ \\
Weight $(\mathrm{kg})$ & 62.6 & 7.9 & $(51.0-81.0)$ \\
SDNN & 56.9 & 18.0 & $(26.8-14.5)$ \\
LF & 637.9 & 430.1 & $(64.0-2239.2)$ \\
HF & 194.0 & 182.3 & $(19.5-1087.4)$ \\
LF/HF ratio & 5.7 & 2.6 & $(1.8-14.4)$ \\
\hline
\end{tabular}


Table II. Mean Value of Pearson's Correlation Coefficient in the Time- and FrequencyDomain Analyses Between 24-hour and 5-minute Recordings

\begin{tabular}{lcc}
\hline & $\begin{array}{c}\text { 24-hour measurement } \\
\text { Mean } \pm \text { SD }\end{array}$ & $\begin{array}{c}\text { Office-hours measurement } \\
\text { Mean } \pm \text { SD }\end{array}$ \\
\hline Time Domain & & $0.668 \pm 0.064$ \\
$\quad$ SDNN versus ASDNN & $0.614 \pm 0.068$ & $0.671 \pm 0.061$ \\
Frequency Domain & & $0.817 \pm 0.064$ \\
LF versus LFm* & $0.578 \pm 0.063$ & $0.562 \pm 0.083$ \\
HF versus HFm & $0.619 \pm 0.069$ & $0.508 \pm 0.062$ \\
RATIO versus RATIOm & & \\
\hline
\end{tabular}

* The subscript " $m$ " indicates variables sampled from the 24-hour measurement.

to those for the time-domain spectra. The correlation of the HRV sampled during office hours was somewhat higher than for the 24-hour measurement.

\section{DISCUSSION}

HRV represents a window on the ability of the heart to respond to internal or external stimuli. The short-term HRV is widely used to assess the variation in the ANS attributable to diverse causes. ${ }^{11,13,14,18)}$ However, little is known about whether the short-term HRV in workers is comparable to 24-hour measurements, which is the standard for assessing the HRV. ${ }^{19)}$ This study measured the 24-hour HRV of workers and evaluated the relationship in time and frequency domain variables, such as SDNN, HF, LF, and LF/HF ratio, between 24-hour and 5minute assessments of the HRV. These variables were widely used to evaluate cardiac autonomic function ${ }^{1,4,9-11,13,14,16)}$ and reflected vagal tone of the SA node or sympathetic and parasympathetic nervous systems. ${ }^{19)}$

We found modest correlations in the time- $(\mathrm{R}=0.614-0.668)$ and frequency$(\mathrm{R}=0.508-0.817)$ domain spectra between the 24-hour and 5-minute HRV. The best correlation between the two was found for the HF spectra $(\mathrm{R}=0.817)$. When restricting the sampling time (09:00-17:00), the correlation between the 24-hour and 5-minute HRV was higher.

Generally, time-domain variables reflect circadian rhythms and the overall change in the ANS. In the frequency domain, the HF spectrum represents parasympathetic nervous modulation of the heart rate, and the LF spectrum reflects a mixture of sympathetic and parasympathetic nervous modulation. Since the HF spectrum is affected by parasympathetic nervous control only, this variation might be relatively minor compared to LF and time-domain indices, such as the 
SDNN and ASDNN, which are regulated by both sympathetic and parasympathetic activity. ${ }^{20)}$ This could explain the high correlation for HF.

A few limitations of this study must be considered when interpreting the results. First, in our study, HRV was measured on 1 day only (24 hour). Therefore, the measured 24-hour HRV might not be representative, as the HRV shows considerable individual day-to-day variation, ${ }^{21,22)}$ although our subjects performed their usual daily work during the test period. Second, we did not measure the 5-minute HRV in a controlled environment; values were selected randomly from the 24-hour recordings. Additionally, our study population was only male, although a sex difference in HRV has been reported. ${ }^{11,23)}$ Therefore, the variation in the 5-minute measurement was large and unstable.

In conclusion, we evaluated the relationship of HRV indices between 24hour and 5-minute recordings. This study demonstrated that the HRV components of a 5-minute recording appear to remain stable and may be applicable for screening the variation in the heart rates of workers, although none of the correlations were strong. Nevertheless, the larger variation or peculiarities of individuals must be considered when the HRV is used to assess the overall status of the ANS. In addition, our results were obtained by examining the relationship between a 24-hour measure and the 5-minute HRV selected from 24-hour recordings, without measuring the 5-minute HRV separately. Therefore, if the 5-minute HRV were obtained under controlled conditions, the correlations might be higher.

\section{REFERENCES}

1. Liao D, Cai J, Rosamond WD, et al. Cardiac autonomic function and incident coronary heart disease: a population-based case-cohort study. The ARIC Study. Atherosclerosis Risk in Communities Study. Am J Epidemiol 1997; 145: 696-706.

2. Malpas SC, Maling TJ. Heart-rate variability and cardiac autonomic function in diabetes. Diabetes 1990; 39: 1177-81.

3. Vaishnav S, Stevenson R, Marchant B, Lagi K, Ranjadayalan K, Timmis AD. Relation between heart rate variability early after acute myocardial infarction and long-term mortality. Am J Cardiol 1994; 73: 653-7.

4. Singh JP, Larson MG, Tsuji H, Evans JC, O'Donnell CJ, Levy D. Reduced heart rate variability and new-onset hypertension: insights into pathogenesis of hypertension: the Framingham Heart Study. Hypertension 1998; 32 : 293-7.

5. Pope CA 3rd, Verrier RL, Lovett EG, et al. Heart rate variability associated with particulate air pollution. Am Heart J 1999; 138: 890-9.

6. Kon H, Nagano M, Tanaka F, Satoh K, Segawa T, Nakamura M. Association of decreased variation of R-R interval and elevated serum C-reactive protein level in a general population in Japan. Int Heart J 2006; 47: 86776.

7. Zuanetti G, Neilson JM, Latini R, Santoro E, Maggioni AP, Ewing DJ. Prognostic significance of heart rate variability in post-myocardial infarction patients in the fibrinolytic era - The GISSI-2 results. Circulation 1996; 94: 432-6.

8. Wichterle D, Simek J, La Rovere MT, Schwartz PJ, Camm AJ, Malik M. Prevalent low-frequency oscillation of heart rate: novel predictor of mortality after myocardial infarction. Circulation 2004; 110: 1183-90. 
9. Pumprla J, Howorka K, Groves D, Chester M, Nolan J. Functional assessment of heart rate variability: physiological basis and practical applications. Int J Cardiol 2002; 84: 1-14. (Review)

10. Migliaro ER, Contreras P. Heart rate variability: short-term studies are as useful as holter to differentiate diabetic patients from healthy subjects. Ann Noninvasive Electrocardiol 2003; 8: 313-20.

11. Liao D, Barnes RW, Chambless LE, Simpson RJ Jr, Sorlie P, Heiss G. Age, race, and sex differences in autonomic cardiac function measured by spectral analysis of heart rate variability--the ARIC study. Atherosclerosis Risk in Communities. Am J Cardiol 1995; 76: 906-12.

12. Goldberger JJ, Challapalli S, Tung R, Parker MA, Kadish AH. Relationship of heart rate variability to parasympathetic effect. Circulation 2001; 103: 1977-83.

13. Lucreziotti S, Gavazzi A, Scelsi L, et al. Five-minute recording of heart rate variability in severe chronic heart failure: correlates with right ventricular function and prognostic implications. Am Heart J 2000; 139: 1088-95.

14. Slaap BR, Boshuisen ML, van Roon AM, den Boer JA. Heart rate variability as predictor of nonresponse to mirtazapine in panic disorder: a preliminary study. Int Clin Psychopharmacol 2002; 17: 69-74.

15. Winsley RJ, Armstrong N, Bywater K, Fawkner SG. Reliability of heart rate variability measures at rest and during light exercise in children. Br J Sports Med 2003; 37: 550-2.

16. Sinnreich R, Kark JD, Friedlander Y, Sapoznikov D, Luria MH. Five minute recordings of heart rate variability for population studies: repeatability and age-sex characteristics. Heart 1998; 80: 156-62.

17. SAS OnlineDoc, Version 8. 1999 SAS Institute Inc. Available at: http://www.asu.edu/sas/sasdoc/sashtml/.

18. Challapalli S, Kadish AH, Horvath G, Goldberger JJ. Differential effects of parasympathetic blockade and parasympathetic withdrawal on heart rate variability. J Cardiovasc Electrophysiol 1999; 10: 1192-9.

19. Task Force of the European Society of Cardiology and the North American Society of Pacing and Electrophysiology. Heart rate variability: standards of measurement, physiological interpretation and clinical use. Circulation 1996; 93: 1043-65.

20. Stein PK, Kleiger RE. Insights from the study of heart rate variability. Annu Rev Med 1999; 50: $249-61$. (Review)

21. Van Hoogenhuyze D, Weinstein N, Martin GJ, et al. Reproducibility and relation to mean heart rate of heart rate variability in normal subjects and in patients with congestive heart failure secondary to coronary artery disease. Am J Cardiol 1991; 68: 1668-76.

22. Kochiadakis GE, Orfanakis AE, Rombola AT, Chrysostomakis SI, Chlouverakis GI, Vardas PE. Reproducibility of time-domain indexes of heart rate variability in patients with vasovagal syncope. Am J Cardiol 1997; 79: $160-5$.

23. Yamasaki Y, Kodama M, Matsuhisa M, et al. Diurnal heart rate variability in healthy subjects: effects of aging and sex difference. Am J Physiol 1996; 271: H303-10. 